\title{
Proceeding
}

Supplementary Issue: Spring Conferences of Sports Science. First International Conference in Iraq on Sport for Peace, 4 April 2019. Baghdad Science Institute, Baghdad, Iraq.

\section{The use of the electrical stimulation device for the muscles in the limit determines the elbow joint during the lifting of the disabled weightlifting players}

\author{
DHAFIR HARB EWAJELA AL-IBRAHEEMI , AHMED MUHAMMAD ABDULKHLIQ ALHASAN, FARAH \\ ISAM ABDULAMEER
}

College of Physical Education and Sports Sciences, University of Baghdad, Iraq

\begin{abstract}
Neuromuscular Electrical Simulation has been improved as the novel therapeutic alternative for the patients who affected with muscle impairment. It has proven significant improvement in strengthening the physical condition and the functional capacity of the patients. In this research work incorporation of Neuromuscular Electrical Simulation for the muscles in the limit determines the elbow joint during the lifting of the disabled weightlifting players. While the weights are lifting by the players without proper cautiousness and concentration the tendon and associated flexor or pronator muscles may get affected and leads to severe pain and causes disability to perform the weightlifting practice. In the process of reviving the weight lifting players different methods are used. The research study focuses on the use of electrical stimulator devices for improving the muscle strength and healing of the elbow joint injuries. Keywords: Weightlifting; Elbow joint injuries; Neuromuscular electrical simulation; Muscle strength improvement.
\end{abstract}

Cite this article as:

Al-lbraheemi, D.H.E., Alhasan, A.M.A., \& Abdulameer, F.I. (2019). The use of the electrical stimulation device for the muscles in the limit determines the elbow joint during the lifting of the disabled weightlifting players. Journal of Human Sport and Exercise, 14(4proc), S488-S496. doi:https://doi.org/10.14198/ihse.2019.14.Proc4.07

Corresponding author. College of Physical Education and Sports Sciences, University of Baghdad, Iraq.

E-mail: Zafer.Atiya@cope.uobaghdad.edu.iq

Supplementary Issue: Spring Conferences of Sports Science. First International Conference in Iraq on Sport for Peace, 4 April 2019. Baghdad Science Institute, Baghdad, Iraq.

JOURNAL OF HUMAN SPORT \& EXERCISE ISSN 1988-5202

(c) Faculty of Education. University of Alicante

doi:10.14198/jhse.2019.14.Proc4.07

S488 | 2019 | Proc4 | VOLUME 14

C 2019 University of Alicante 


\section{INTRODUCTION}

Injuries are commonly encountered in weightlifting practices. The most common injuries and muscle loss can be identified are with the tennis elbow or lateral epicondylitis, Shoulder Hurts. This condition gives raise to the pain when the weight is lifted. This condition defunct the practice of the player. When the player did the practice of weightlifting with less concentration this condition can be experienced by the player (Proske \& Gandevia,2012). It causes the inflammation in the tendon muscles and increases the pain in the elbow joint and the forearm muscles. The pain can crawl to the other parts of the hand like wrist and fingers. The study of Rheumatology says that the Tennis Elbow is common for the people who are involved with the lots of arm and writs movements. Weightlifting players are commonly performing the exercise which is four times greater than normal work done by ordinary human beings (Baudisch\& Naumann, 2018). Tennis Elbow gradually worsen the performance of the player and makes them weak. The part of the elbow joint sometimes swollen and pain spread across the forearm when the player stretches the hand. According to the journal of sports health, further extended complications can be suppressed by consulting a doctor and start the physical therapy. Rotator Cuff and Shoulder Warm-up is another remedy to reduce the further complexities caused by the tennis elbow (Velloso et al, 2013). Stimulating devices are providing the betterment for the muscle strength and reduce the pains and further complications to the players with its significant quality of performance. The usage of stimulating devices can enable the user to operate the machine with great use. It is a proven fact that the electrical stimulating devices have provided the improvement of muscles strength and relief from the pain (Barber-Westin \& Frank, 2017). The electrical stimulation has provided the condition to have the normal living function and gradually provided the strength to perform the weightlifting practices (Kennedy, 2011). The electrical muscle stimulation devices are used as therapeutic agents. The use of this muscle stimulation devices are giving rise to the caloric expenditure and promote weight loss also. Muscle Stimulation is a latest technique which can reduce the frequent visits to the clinics and allow the patients dramatically improve the effectiveness of therapy. Muscle Stimulation Devices can be used by the patients at home and recover from the muscle loss in the very early stages (Rehabilitation of Primary, 2017). The main purpose of the Electrical Muscle Stimulators is to provide the rehabilitation to the neurological impairments with the help of exoskeleton treatment. The cost of the devices are high and barrier to the patient to keep the devices at home and have the treatment. The portable devices are promoted in the market but the cost still needs to be reduced to have more popularity and usability by the individuals (Estevea et al., 2019). The neuromuscular electrical stimulation is a process performed with the electric impulses. This is basically providing the strength accumulation to the muscles with is gradual training for the athletes. This is also used as a testing tool to evaluate the neural and muscular functionality required by them to do practice. The main purpose of the Neuromuscular Electrical Stimulation tools is to be used as post exercise recovery tool (Kono et al., 2018). This tool is connected with the electrodes which can be attached or placed near the muscle with the help of pads. The electrodes attached to the muscles will stimulate the central nervous system and improve the condition with the help of potential impulses (Pohl et al., 2018). These devices are widely used by the sports scientists as a complementary technique in the weightlifting champions training centres. These devices have been approved by US Food and Drug Administration [FDA] (Gómez et al., 2017).

\section{METHODOLOGY}

\section{Electrical Muscle Stimulation}

Electrical Muscle Stimulation or neuromuscular electrical stimulation is the same. The device consists of two types of parts which are connected with the wires. These are Electrical Muscle Stimulation apparatus which 
display the results and training process. The other part is pads. These pads are connected to the training apparatus (Gonzalez et al., 2018).

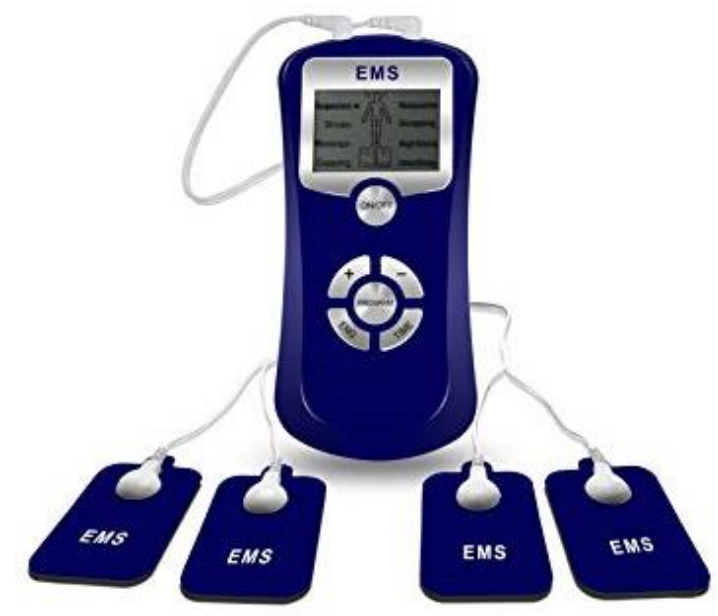

Figure1. EMS

\section{Simulating Impacts}

The weightlifting players are getting frequent muscle impairment with the cause of Tennis Elbow. The extensor muscle is connected to the common extensor tendon. When the common extensor tendon is affected automatically it gives raise to the pain and defunct of the practice. If this is left like that it increase the pain to the wrists and figure joints (Lopes, 2018).

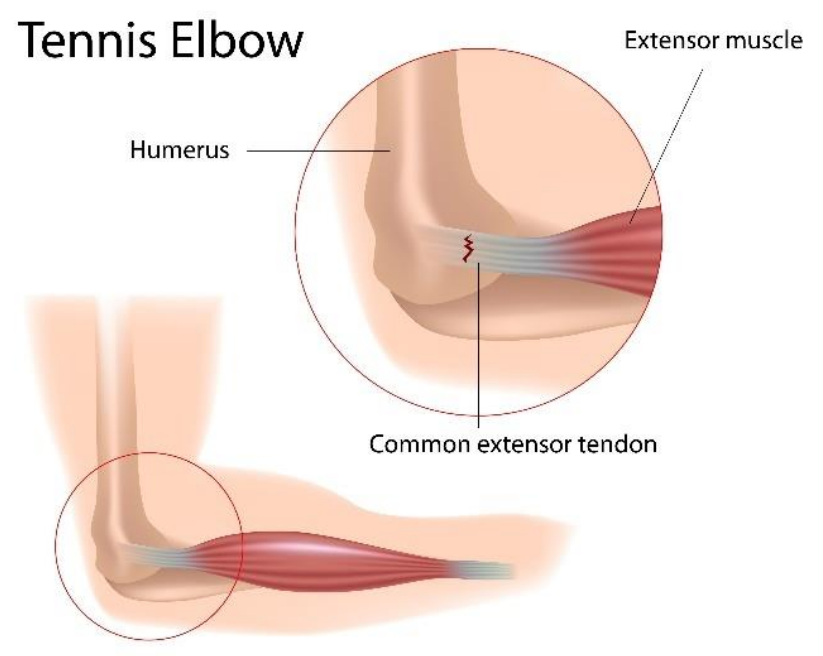

Right arm, lateral (outside) side

Figure 2. Muscle Impairment and Injury

The doctors suggest immediate treatment for the muscle impairment happened at common extensor tendon. The condition can be spread to the forearm flexor muscles, bone associated with the muscles and finger joints in association with the wrists joint and Humerus also. The injured common extensor tendon needs to 
be revived and recover from the condition. The most suitable treatment to recover this situation with the help of EMS is preferably suggested (Lopes, 2018).

\section{Theory of Concept}

EMS is the device which is acting on the central nervous system to recover the muscle impairment. The injury is identified in the tendon will affect the muscle at the back of upper arm to the bony bump which is situated at the back of the elbow (Kamihira et al., 2016). EMS can be used effectively to cure the strain, tendinitis. The function of the device is to stimulate the central nerves system to reduce the Muscle soreness, revive scar tissue, heal soft tissue, improve the blood circulation in the arm and rehabilitate the arm strength to the original state of functioning (Lewin, 2018).

The revival process can take 60 to several hours for a period of 3 to 6 days. Based on the injury intensity the treatment and training needs to provide to the arm to stimulate the central nervous system on day to day basis for a stipulated time frame of 10 to 60 minutes a day. The use of EMS can be done 1 time to 3 times a day. It should be implemented under the observation of a doctor or scientist (Copaci et al., 2017).

The functionality of the EMS can be divided in two levels. One is at the wrist module. The second is at Elbow Module. EMS electrodes can be placed at Elbow Module and Wrist module. The modules are associated with the wrist motor and Elbow Motor. These electrodes can stimulate the central nervous system to revive the condition at Elbow and Wrist. These two parts are predominantly affected by the muscle impairment. Hence these modules need to be concentrated. The computer or EMS device is connected to Wrist Motor and Elbow Motor. These modules will continuously send the stimulating signals to the body and receives the response from these modules. The condition of the muscles can be demonstrated and recorded in the reading in the computer monitor or EMS device. The time duration can be judged according to the improvement recorded in the monitor. This may lead to 10 minutes to 60 minutes accumulated from the patient body and decision of the doctor (Soltani-Zarrin et al., 2016).

The main functionality of the device is generated from two modules. These modules are supported by hanging rail, hanging spring. But in the newest devices these are eliminated and connected with only two Electrodes covered by pads. This machine receives the joint angular velocity from the body and records the betterment condition of the muscles. The readings can be viewed through the connected monitor or device screen (Alrwaily, 2017). The proposed EMS machine can send the impulses from the device and receives the signal from the body and demonstrate the results (Hayter et al., 2005).

EMS is basically sending signal or impulses to the body and stimulate the central nervous system to get revive of the muscle impairments. In this treatment the doctors can determines the limitation of the muscle strength and improve the condition of the patient according to the sustainable capacity of the patient. In this treatment the severity of the injury is also playing vital role in determining the period of treatment and intervals to apply the treatment per day (Eraifej et al., 2017). The latest devices available in the market has become viable for the patients also. The patients should have the knowledge to apply the device and stimulate the muscle strength (Sousa et al., 2018). The devices are mostly applied and used by the doctors in the athletes' rehabilitation centres only (Shaw et al., 2015). 
a

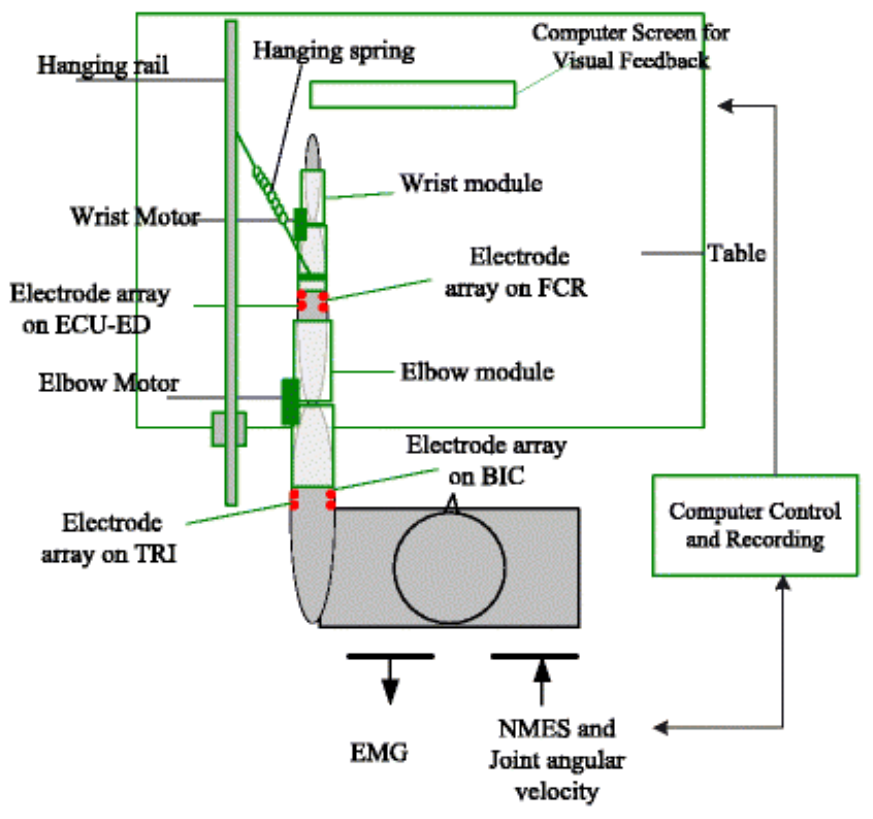

b

d
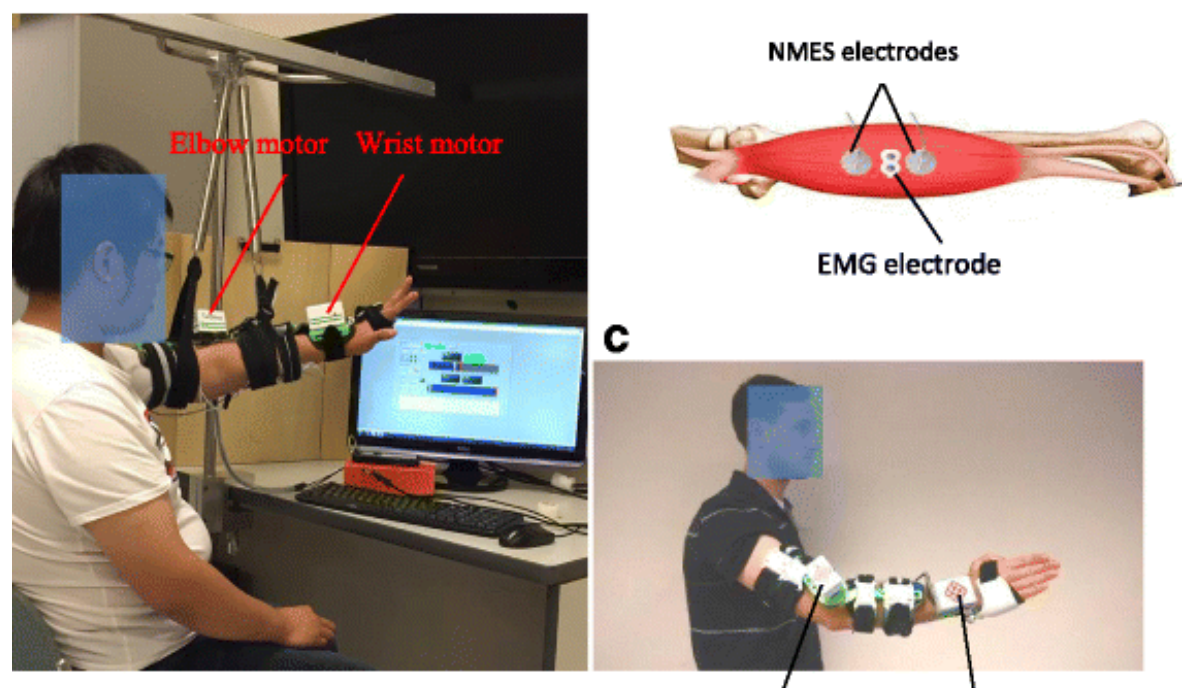

C

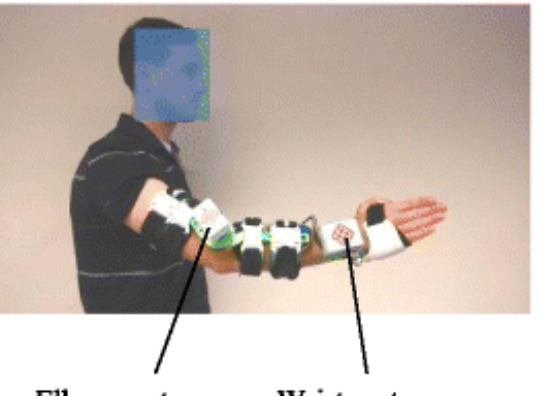

Elbow motor Wrist motor

Figure 3. Functionality of EMS

\section{The solution}

When the weightlifting players are affected with the muscle impairments it is suggested to apply the Electrical Simulators to revive them from the skeletor muscle impairment. The solution can be given from EMS for not only for the muscles but also the affected bones (MOR et al., 2017). 


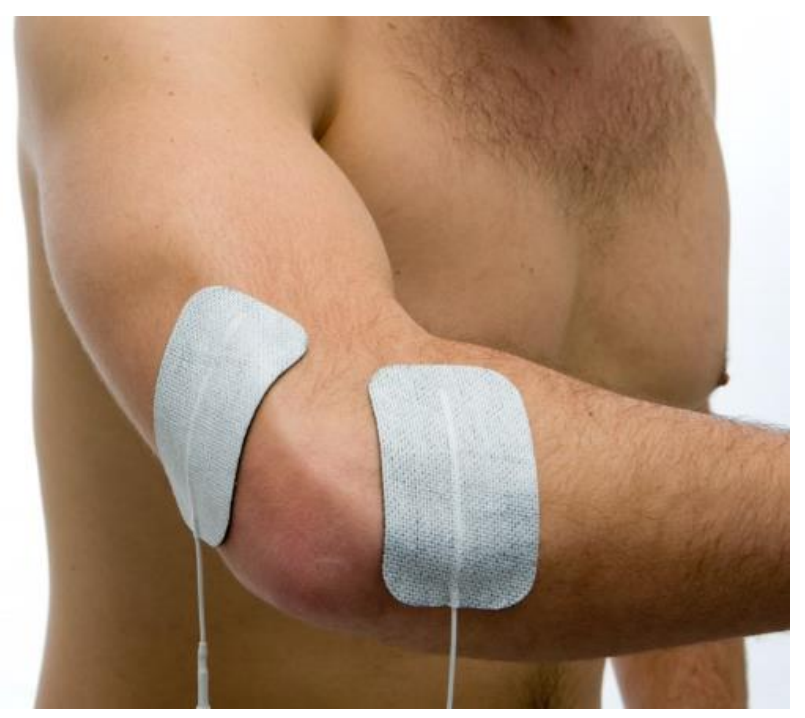

Figure 4. Treatment with EMS

When the bone is affected with remarkable crack or scar. It needs more time to revive rather than the muscle recovery. The treatment procedures can be practiced from the doctor and needs to be done by the patient on their own. But it is always suggested that the strict supervision of doctor is suggested. The following table can reveal the time frame and frequency of usage of EMS in healing the impairment of the skeletal muscles (Esteve et al., 2017).

Table1. Intensity of usage of EMS

\begin{tabular}{|c|c|c|}
\hline Purpose & Intensity & Frequency \\
\hline Muscle Pain relief & Low & One time \\
\hline Soreness in Muscle & Low & 1 or 2 Times \\
\hline Tissue Scar & Low & 1 time \\
\hline Soft Tissue & Medium & 3 times \\
\hline Blood Circulation & Low & 2 times \\
\hline Skeleton scar & High & 2 times \\
\hline
\end{tabular}

When the intensity is low the treatment with the system can be used with limited time from 10 to 30 minutes can be given. If the intensity is medium the treatment can be provided to the patient from 30 to 60 minutes. When the intensity is high it needs to provide the treatment with fixed small intervals for several hours a day (Health Quality Ontario, 2017).

The electrode pads should be attached to both hands to justify the strength of the affected hand should be reached to the normal hand strength. The device reading should be compared with two hands and the device will suggest the treatment and impulses should continue (Fateminasab, 2014). The treatment should continue until the affected hand reaches the state and strength of normal hand. The proposed and available EMS devices are providing with more electrodes to examine two hands at a time and compare the strength of the muscles of two hands. This operation can give proven results and revival from the elbow joint injuries while doing the practice of weightlifting (Sachetti et al., 2018). 


\section{CONCLUSIONS}

The use of the electrical stimulation device for the muscles in the elbow joint is the proposed research topic. In this topic the Neuromuscular Electrical Simulation has been explained to it improves the strength of the affected muscle. The latest advancements in the scientific development in the field of clinical biology equipment has provided the Neuromuscular Electrical Simulation equipment. It has provided results in strengthening the physical condition and the functional capacity of the patients. In this research work the functionality of the Neuromuscular Electrical Simulation device is explained and how it should be used for the patients affected with the derailment of the elbow joints in weightlifting precise. In the process of reviving the weightlifting players different methods are used. The research study focused properly on the use of electrical stimulator devices for improving the muscle strength and healing of the elbow joint injuries for weightlifting players.

\section{REFERENCES}

Uwe Proske, and Simon C. Gandevia. (2012). The Proprioceptive Senses: Their Roles in Signaling Body Shape, Body Position and Movement, and Muscle Force. https://doi.org/10.1152/physrev.00048.2011

Patrick Baudisch and Felix Naumann. (2018). interactive systems based on electrical muscle stimulation published by Institutional Repository of the University of Potsdam.

Eduardo Velloso, Andreas Bulling, Hans Gellersen, Wallace Ugulino,Hugo Fuks.(2013).Qualitative activity recognition of weight lifting exercises published in $\mathrm{AH}$ '13 Proceedings of the 4th Augmented Human International Conference Stuttgart, Germany - March 07 - 08, 2013 ACM New York, NY, USA @2013URN urn:nbn:de:kobv:517-opus4-421165. https://doi.org/10.1145/2459236.2459256

Sue D. Barber-Westin, Frank R. (2017). Rehabilitation After Anterior Cruciate Ligament Autogenous Reconstruction Published in Noyes' Knee Disorders: Surgery, Rehabilitation, Clinical Outcomes (Second Edition), Science Direct. https://doi.org/10.1016/b978-0-323-32903-3.00010-x

Carol Kennedy. (2011). Therapeutic exercise for mechanical neck pain published in Science Direct Neck and Arm Pain Syndromes. https://doi.org/10.1016/b978-0-7020-3528-9.00013-3

Rehabilitation of Primary and Revision Anterior Cruciate Ligament Reconstruction published in Noyes' Knee Disorders: Surgery, Rehabilitation, Clinical Outcomes (Second Edition), ( 2017). Science Direct. https://doi.org/10.1016/b978-0-323-32903-3.00008-1

Vicent Estevea, José Carneirob, Fátima Moreno, Miquel Fulquet, Salud Garrigac, Mónica Poub, Verónica Duarteb, Anna Saurinab, Irati Tapiab, Manel Ramírez de Arellano .(2019) .The effect of neuromuscular electrical stimulation on muscle strength, functional capacity and body composition in haemodialysis patients published in elsevier 2019 Journal of Electromyography and Kinesiology. https://doi.org/10.1016/j.nefroe.2017.01.011

Michinari Kono,Yoshio Ishiguro, Nagoya , Takashi Miyaki and Jun Rekimoto .(2018) .Design and Study of a Multi-Channel Electrical Muscle Stimulation Toolkit for Human Augmentation published in Proceeding $\mathrm{AH}$ '18 Proceedings of the 9th Augmented Human International Conference Article No. 11. https://doi.org/10.1145/3174910.3174913

Henning Pohl, Kasper Hornbæk and Jarrod Knibbe. (2018) .Wanding Through Space: Interactive Calibration for Electric Muscle Stimulation published in 2018 Copyright held by the owner/author(s). Publication rights licensed to Association for Computing Machinery. ACM ISBN 978-1-4503-54158/18/02. https://doi.org/10.1145/3174910.3174948

Cuesta-Gómez, Alicia \& Molina-Rueda, Francisco \& Carratalá-Tejada, María \& Imatz Ojanguren, Eukene \& Torricelli, Diego \& Page, juan-carlos. (2017). The Use of Functional Electrical Stimulation on the 
Upper Limb and Inter-scapular Muscles of Patients with Stroke for the Improvement of Reaching Movements: A Feasibility Study. Frontiers in Neurology. 8. 10.3389/fneur.2017.00186.AH2018, February 7-9, 2018, Seoul, Frontiers in Neurology Republic of Korea. https://doi.org/10.3389/fneur.2017.00186

Eric J. Gonzalez , Ryan J. Downey, Courtney A. Rouse, and Warren E. Dixon. (2018). Influence of Elbow Flexion and Stimulation Site on Neuromuscular Electrical Stimulation of the Biceps Brachii IEEE Transactions on neural systems and rehabilitation engineering, vol. 26, no. 4. https://doi.org/10.1109/tnsre.2018.2807762

Pedro Lopes .(2018). interactive systems based on electrical muscle stimulation published in Creative Commons International.

Rintaro Kamihira, Misato Kasuya, Yinlai Jiang, Shunta Togo, Masao Sugi, Hiroshi Yokoi .(2016).Maintenance of Muscle Contraction Improves during Dynamic Exercise by Multi-channel Functional Electrical Stimulation with Time Shifting Stimulation published in International Journal of Bioscience, Biochemistry and Bioinformatics. https://doi.org/10.17706/ijbbb.2017.7.1.33-40

Bernard Lewin .(2018). Functional Electrical Stimulation and Neuromuscular Electrical Stimulation published in Clinical Policy Bulletin published in Aetna Better Health of Pennsylvania.

Dorin Copaci, Enrique Cano, Luis Moreno, and Dolores Blanco. (2017). New Design of a Soft Robotics Wearable Elbow Exoskeleton Based on Shape Memory Alloy Wire Actuators published in Applied Bionics and Biomechanics Volume 2017, Article ID 1605101, 11 pages. https://doi.org/10.1155/2017/1605101

Rana Soltani-Zarrin, Amin Zeiaee, Reza Langari, Reza Tafreshi .(2016). Challenges and Opportunities in Exoskeleton-based Rehabilitation published in Springer NSF grant 1644743 and QNRF, NPRP 71685-2-626.

Muhammad Alrwaily .(2017). A Comparison Between Stabilization Exercises and Stabilization Exercises Supplemented with Neuromuscular Electrical Stimulation in Patients With Chronic Low Back Pain: A Phase I Randomized Controlled Trial published by university of Pittsburgh. https://doi.org/10.1016/i.bjpt.2018.10.003

Tina L. Hayter, Jeff S. Coombes, Wade L. Knez, And Tania L. Brancato .(2005). Effects Of Electrical Muscle Stimulation On Oxygen Consumption published in Journal of Strength and Conditioning Research, 2005, 19(1), 98-101 2005 National Strength \& Conditioning Association. https://doi.org/10.1519/00124278-200502000-00017

John Eraifej, William Clark, Benjamin France, Sebastian Desando and David Moore .(2017).Effectiveness of upper limb functional electrical stimulation after stroke for the improvement of activities of daily living and motor function: a systematic review and meta-analysis published in Systematic Reviews .(2017). 6:40 Creative Commons Attribution 4.0 International License of Open Access. https://doi.org/10.1186/s13643-017-0435-5

Davide Gde Sousa, Lisa AHarvey, Simone Dorsch and Joanne VGlinsky .(2018). Interventions involving repetitive practice improve strength after stroke: a systematic review published in Journal of Physiotherapy Volume 64, Issue 4, October 2018, Pages 210-221. https://doi.org/10.1016/i.jphys.2018.08.004

Brandon S Shaw, Gregory A Brown and Ina Shaw .(2015). Resistance exercise is medicine: Strength training in health promotion and rehabilitation published in International Journal of Therapy and Rehabilitation 22(8):385-389. https://doi.org/10.12968/ijtr.2015.22.8.385

Ahmet Mor, Gökhan Ipekoğlu, Cansel Arslanoğlu, Kürşat Acar,Erkal Arslanoğlu .(2017). Effects of Some Active and Passive Recovery Techniques on Strength Parameters published in International Journal of Science Culture and Sport Research artcle published on 29-08-2017. 
Vicent Esteve, José Carneiro, Fátima Moreno, Miquel Fulquet, Salud Garriga, Mónica .(2017). The effect of neuromuscular electrical stimulation on muscle strength, functional capacity and body composition in haemodialysis patients Published in Elsevier Sociedad Española de Nefrologían e f r o l o g i a. 2 0 1 7;3 7(1):68-77. https://doi.org/10.1016/j.nefroe.2017.01.011

Health Quality Ontario. (2017). Electrical Stimulation for Pressure Injuries: A Health Technology Assessment published in Ontario Health Technology Assessment Series. 2017; 17(14): 1-106. Published online 2017 Nov 8.PMCID: PMC5700239.

Amanda Sachetti, Marta Fiorvanti Carpes, Alexandre Simões Dias, Graciele Sbruzzi .(2018). Safety of neuromuscular electrical stimulation among critically ill patients: systematic review published in SciELO Analytics Scientific Electronic Library Online vol.30 no.2 São Paulo Apr./June 201800. https://doi.org/10.5935/0103-507x.20180036

Ali, Fateminasab. (2014). Investigating the challenges and barriers of convergence between Iran and republic of Azerbaijan, UCT Journal of Social Sciences and Humanities Research, Issue 1,pp.18-24.

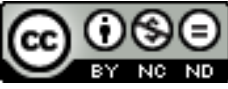

This work is licensed under a Attribution-NonCommercial-NoDerivatives 4.0 International (CC BY-NC-ND 4.0). 